\title{
Harmonic Distortion Caused by Multilevel Current Source Inverters
}

\author{
S. Koenig ${ }^{1}$ and G. Herold ${ }^{1}$ \\ ${ }^{1}$ Chair of Electrical Energy Systems \\ University of Erlangen-Nuremberg \\ 91058 Erlangen (Germany) \\ Phone number: +49 9131 85-29526, e-mail: Simon.Koenig@fau.de
}

\begin{abstract}
Due to power electronic reasons and the cheaper construction possibilities of the link capacitors, the voltage source based topology is nowadays the state of the art. With the multilevel possibility the next step in inverter technology was taken and again the professional discussion mainly focusses on the voltage source inverters. This paper shall explain the approach of using a multilevel current source inverter instead.
\end{abstract}

\section{Key words}

Multilevel Current Source Inverter, Harmonics, Power Quality.

\section{Introduction}

Regarding current developments in the area of high voltage high power inverter applications the main movement is to increase the number of levels. There are two main reasons for that. First it is possible to significantly decrease the stress for the power electronic equipment, secondly the caused distortion in the output variables are distinctly smaller [1].

\section{Basic Requirements for CSI Switching Schemes}

\section{A. Space Phasor Representation of CSI}

Fig. 1 shows the equivalent space phasor network of a current source topology (multilevel or standard). The current source $\underline{i}_{c s i}$ represents the power electronic part including the dc source and the link element, an inductance in this case. This is only valid if the following assumptions are met. On the one hand the control has to adequately balance the different dc currents in a multilevel case, and on the other hand the replacement of the power semiconductors with ideal switches has to be properly legitimated for the considered research topic.

The network also includes the necessary output capacitors, which are obligatory due to the fact, that fast changing output currents would cause extreme overvoltages at the grid inductivities if connected directly [2].

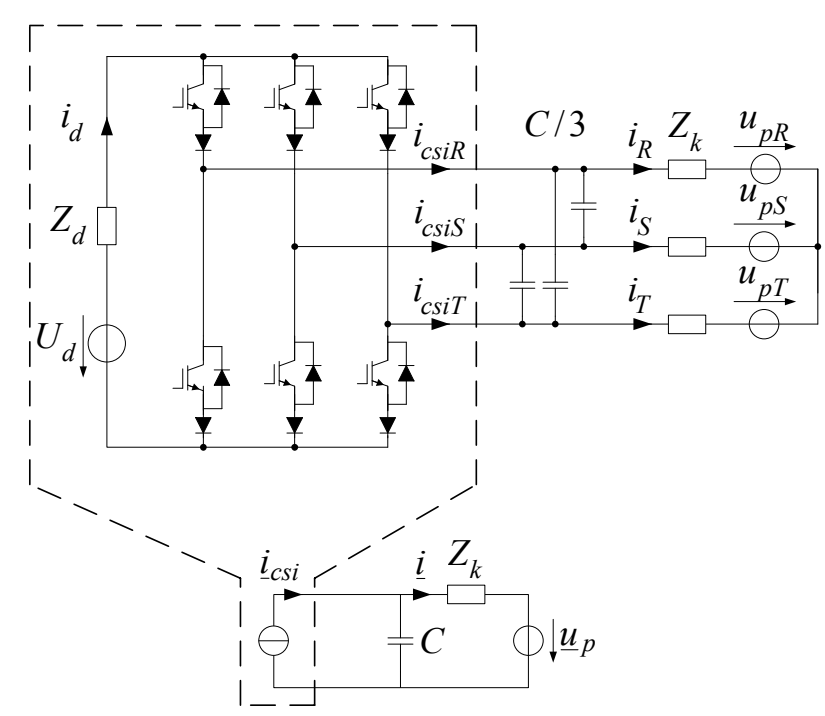

Fig. 1. Three phase and equivalent space phasor network

The space phasor and the zero sequence of every network variable can be obtained with the following formulas

$$
\begin{aligned}
& \underline{v}=\frac{2}{3}\left(v_{R}+\underline{a} v_{S}+\underline{a}^{2} v_{T}\right) \quad \text { with } \quad \underline{a}=\mathrm{e}^{\mathrm{j} \frac{2 \pi}{3}} \\
& v_{0}=\frac{1}{3}\left(v_{R}+v_{S}+v_{T}\right)
\end{aligned}
$$

\section{B. Passive Network Behaviour}

The effects of the passive network on the CSI output currents can best be judged using transfer functions. The grid current $\underline{i}$ is connected with the inverters output current $\underline{i}_{c s i}$ by (3), whereas (4) shows the relation between the harmonics of the capacitor voltage and the CSI output current space phasor.

$$
\begin{aligned}
& \underline{G}_{c s i}=\frac{\underline{\hat{I}}_{n}}{\underline{\hat{I}}_{c s i, n}}=\frac{1}{1-\omega^{2} L_{k} C+\mathrm{j} \omega R_{k} C} \\
& \underline{G}_{c s i U}=\frac{\underline{\hat{U}}_{c, n}}{\underline{\hat{I}}_{c s i, n}}=\underline{Z}_{c} \| \underline{Z}_{k}=\frac{R_{k}+\mathrm{j} \omega L_{k}}{1-\omega^{2} L_{k} C+\mathrm{j} \omega R_{k} C}
\end{aligned}
$$

The network shows resonant behaviour (no imaginary part of the network impedance in (4)) for 


$$
f_{\text {res } U}=\frac{1}{2 \pi} \sqrt{\frac{1}{L_{k} C}-\frac{R_{k}^{2}}{L_{k}^{2}}}=f_{0} \sqrt{1-\frac{R_{k}^{2} C}{L_{k}}}
$$

where $f_{0}$ is the resonance of the ideal lossless parallel resonant circuit. Following (3) the frequency at which $\underline{G}_{c s i}$ shows the maximum amplification, is

$$
f_{\text {res }}=\frac{1}{2 \pi} \sqrt{\frac{1}{L_{k} C}+\frac{R_{k}^{2}}{2 L_{k}^{2}}}=f_{0} \sqrt{1+\frac{R_{k}^{2} C}{2 L_{k}}}
$$

The grid current leaves this resonance area again for frequencies higher than (cf. Fig. 2)

$$
f_{\text {damp }}=\frac{1}{2 \pi} \sqrt{\frac{2}{L_{k} C}-\frac{R_{k}^{2}}{L_{k}^{2}}}=f_{0} \sqrt{2-\frac{R_{k}^{2} C}{L_{k}}}
$$

In weakly damped networks $\left(\varphi_{k} \approx \pi / 2 \Rightarrow R_{k} \approx 0\right)$ all three resonance frequencies become approximately equal

$$
f_{\text {resU }} \approx f_{\text {res }} \approx f_{0}=\frac{1}{2 \pi \sqrt{L_{k} C}}
$$

As a consequence it is for a given network reactance $L_{k}$ possible to achieve a desired resonance frequency by choosing the adequate capacitor value following formula (8). Finally Fig. 2 shows the bode plot of the transfer function $\underline{G}_{c s i}$ of (3) for the space phasor network parameters given in Table 1.

Table 1: Network Parameters

$$
\begin{array}{c|c|c}
R_{k} \text { in } \mathrm{m} \Omega & L_{k} \text { in } \mu \mathrm{H} & C \text { in } \mathrm{mF} \\
\hline 2.65 & 47.77 & 5.89
\end{array}
$$

It can be seen, that the chosen capacitance leads to a resonance frequency of $300 \mathrm{Hertz}$. The resulting resonance limit frequency $f_{\text {damp }}$ is about $\sqrt{2} f_{0}=424$ Hertz.

$$
\text { - } f_{0} \times f_{\text {damp }}
$$

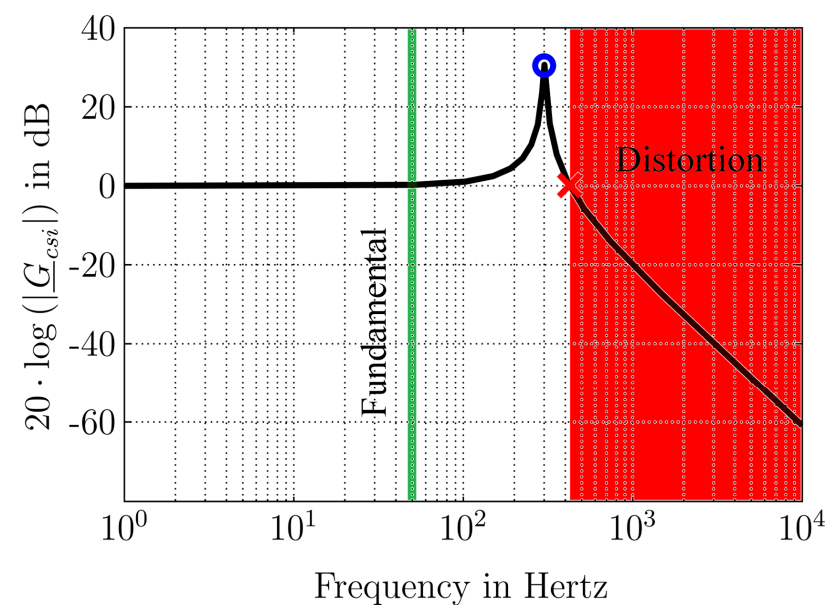

Fig. 2: Transfer function $\underline{G}_{c s i}$

\section{Switching Schemes}

Furthermore Fig. 2 clearly indicates the required properties of the chosen switching scheme. Its first harmonic distortion should not occur in the resonance area below $f_{\text {damp. }}$. Discussing the possible use of multilevel inverters the question arises in which manner this increase in levels affects the resulting spectrum of the inverters output current in comparison to the standard topology of Fig. 1 containing three level output currents.

\section{Multilevel Current Source Inverters}

\section{A. From standard to multilevel topology}

Fig. 3 demonstrates the principle of the additional levels in the space phasor diagram. The black points represent the possible states of a three level inverter, the blue ones those of a five level inverter. The level count in this paper includes the negative levels.

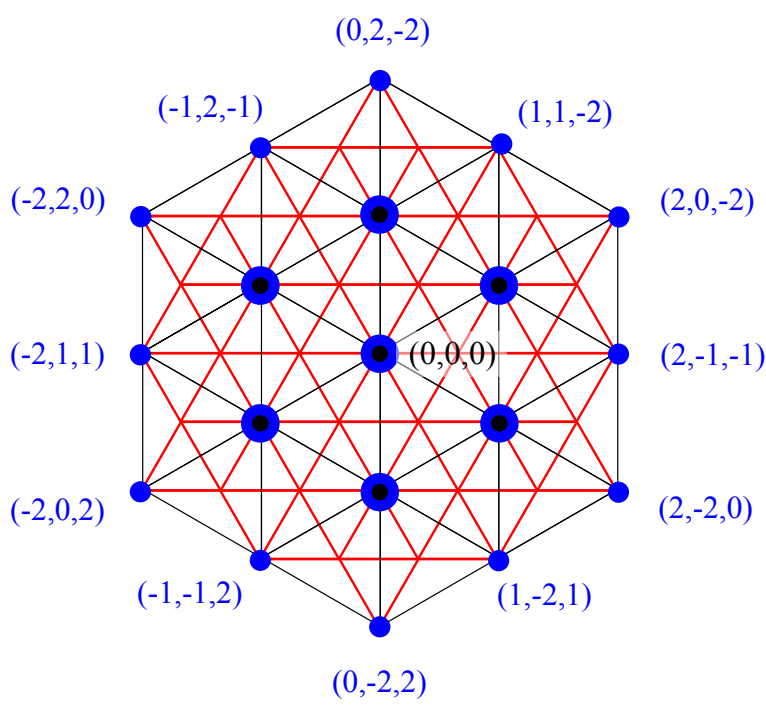

Fig. 3. Possible states in the complex plain of a three (black) and a five (blue) level CSI

Topological the five level inverter could for example be achieved by paralleling two three level inverters [3]. In operation one then must assure, that both inverters provide the same direct current. This principle can theoretical be extrapolated to any uneven level number. Mention, that the space phasor equivalent network of Fig. 1 is also valid for every multilevel topology.

There are two significant differences between the CSI and the voltage source inverter (VSI) space phasor diagram. First the idea of following the state-connectionlines to count the necessary line quantities is not possible because of the demand, that the output zero sequence current of the CSI has to be zero for every moment.

$$
i_{c s i 0}=0 \quad \forall t
$$

Therefore Fig. 3 also contains the necessary "walking paths" for the CSI to reach the states in red.

As a direct consequence the states of the line current $i_{c s i R}$ can be directly seen by projecting the current complex space phasor state on the real axis of the complex plane.

$$
i_{c s i R}=\operatorname{Re}\left\{\underline{i}_{c s i}\right\}+i_{c s i 0}=\operatorname{Re}\left\{\underline{i}_{c s i}\right\}
$$

Due to the operation principle of six-pulse inverters their lowest order distortion starts with the $-5^{\text {th }}$ and the $+7^{\text {th }}$ harmonic in the space phasor spectrum. Referring to Fig. 2 and equation (7) that means, that those two harmonics would appear in the resonance area and therefore would 
cause resonance amplifications in the capacitor voltage $\underline{u}_{c}$ and the grid current $\underline{i}_{c s i}$ of Fig. 1. Consequently the outputspectrum of the inverter should not contain significant $-5^{\text {th }}$ and $+7^{\text {th }}$ harmonics. Further harmonics like the $-11^{\text {th }}$ and the $+13^{\text {th }}$ are already out of the resonance and do not represent a problem. To be able to allocate the occurring distortion the total harmonic distortion

$$
\begin{gathered}
\mathrm{THD}=\frac{\sqrt{\sum_{n=2}^{\infty} \hat{V}_{n}^{2}}}{\hat{V}_{1}} \cdot 100 \% \\
\mathrm{THD}_{40}=\frac{\sqrt{\sum_{n=2}^{40} \hat{V}_{n}^{2}}}{\hat{V}_{1}} \cdot 100 \%
\end{gathered}
$$

and the $\mathrm{THD}_{40}$ which only represents the distortion up to the $40^{\text {th }}$ harmonic are introduced.

\section{B. Fundamental Frequency Modulation (FFM)}

The results of this section are obtained by a fundamental frequency switching scheme introduced for VSI in [4]. In comparison to high frequency switching schemes like the pulse width modulation (PWM) and the space vector pulse width modulation (SVPWM) each space phasor state is only used once in a fundamental frequency period. This switching scheme was adopted for CSI and therefore allows the calculation of the harmonic behaviour of the inverters output space phasor. The principle evaluation can be seen in Fig. 4.

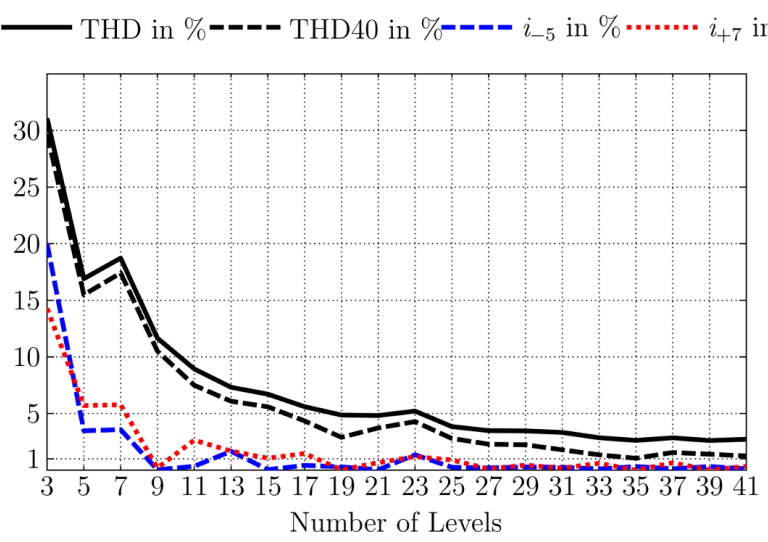

Fig. 4. THD, occurrence of 5 th and 7 th harmonic for fundamental frequency switching scheme

It shows the THD, the limited $\mathrm{THD}_{40}$ and the $-5^{\text {th }}$ and $7^{\text {th }}$ harmonic related to the fundamental over different level numbers. The low order harmonics are rapidly decreasing by the number of levels but not quite predictable. For more than 25 levels they stay below $1 \%$ of the fundamental. The THD, starting at about $30 \%$, is also decreasing fast and nearly equal to the $\mathrm{THD}_{40}$. That means that the occurring distortion mostly takes place below the $40^{\text {th }}$ harmonic. For theoretical high level numbers it reaches about $3 \%$. Fig. 5 shows the harmonic content without the fundamental for a 3 and a 41 level CSI more in detail until the $40^{\text {th }}$ harmonic. They are related to the fundamental.

$$
i_{n}=\hat{I}_{n} / \hat{I}_{1}
$$

The effect of the level increase becomes quite obvious. The harmonics are all reduced to a very low similar level.

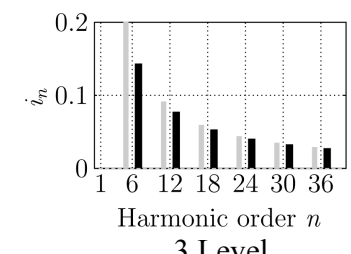

3 Level

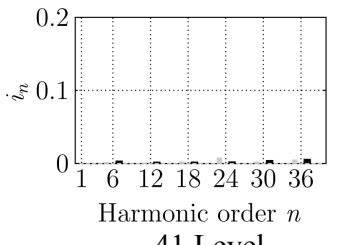

41 Level
Fig. 5: FFM distortion spectra for 3 and 41 level CSI

\section{Space Vector Pulse Width Modulation Switching Scheme}

In comparison to the previous section, now a space vector pulse width modulation is used. It is explained more in detail in [5] for VSI and again adapted for CSI. The basic principle is to choose a certain number of discrete and equidistant points of the desired fundamental frequency space phasor. Those points are allocated in the belonging triangles, cf. Fig. 3, and finally the switch on times of the adjacent states are calculated.

Fig. 6 shows the results for 24 target points. There are two main differences in comparison to the FFM.

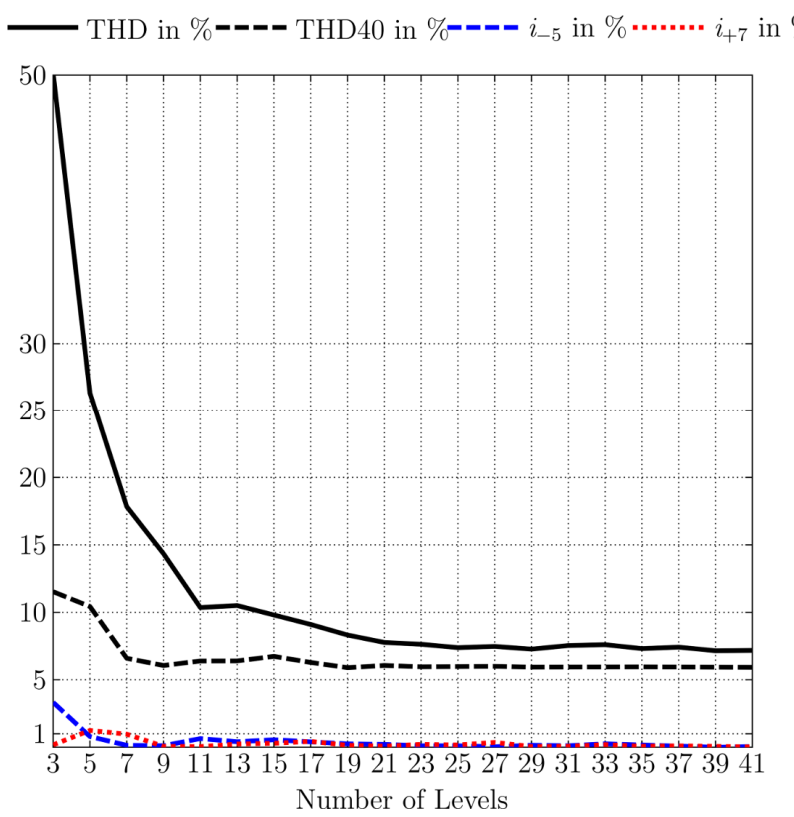

Fig. 6: THD, occurrence of 5th and 7th harmonic for space vector modulation switching scheme reproducing 24 target points

First the THD is much higher at the beginning and only decreases to about $7 \%$. Until around 15, 17 levels there is a significant difference between the THD and the $\mathrm{THD}_{40}$. For higher levels the main distortion again takes place below the $40^{\text {th }}$ harmonic. Furthermore the low order harmonics can already be kept below $1 \%$ for levels higher than 9. This can be confirmed by Fig. 7. For a low level number the output contains all six-pulse harmonics, beginning with the $-5^{\text {th }}$. On the other hand an inverter 
with a high level number nearly only contains the $-23^{\text {th }}$ and $+25^{\text {th }}$ harmonic anymore.

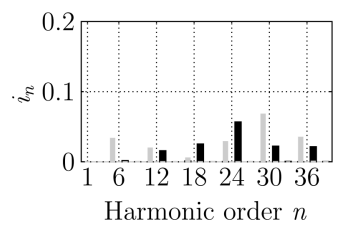

3 Level

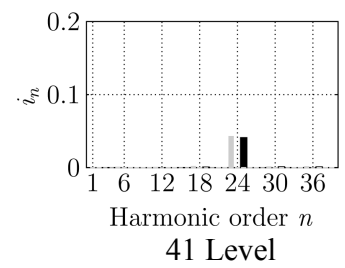

Fig. 7: SVPWM distortion spectra for 3 and 41 level CSI, 24 target points

Fig. 8 now displays the curve progressions for a SVPWM reproducing 48 target points. The low order harmonics can be kept below $1 \%$ for each multilevel topology. The $\mathrm{THD}_{40}$ now clearly deviates from the THD due to the fact, that the 48 target points lead to a distortion centre around the $48^{\text {th }}$ harmonic, thus the $-47^{\text {th }}$ and the $+49^{\text {th }}$ harmonic which are not included in the THD 40 .

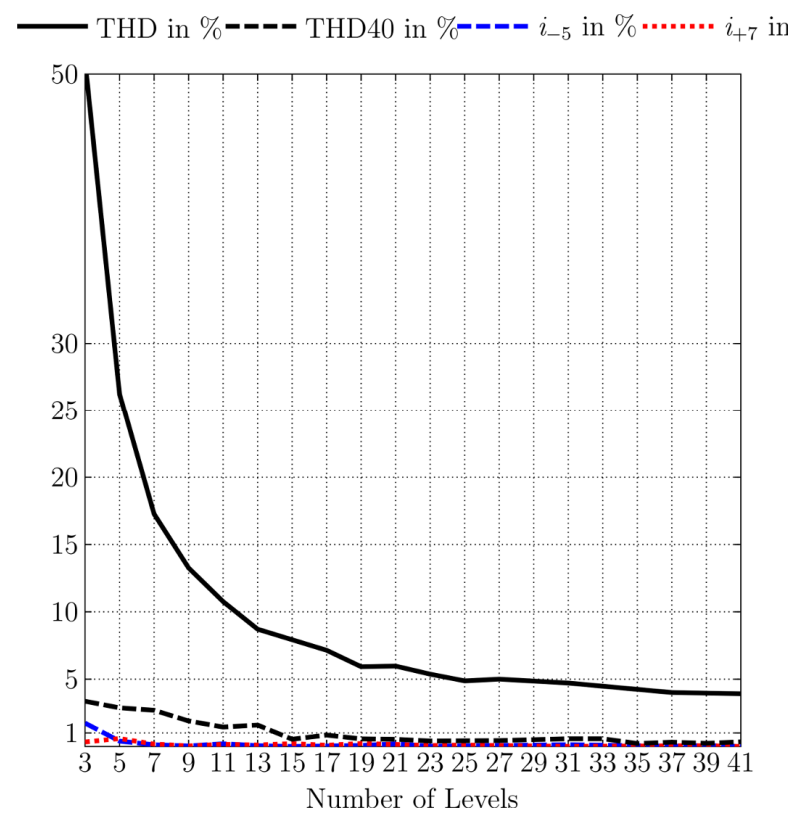

Fig. 8: THD, occurrence of 5th and 7th harmonic for space vector modulation switching scheme reproducing 48 target points

These findings can also be seen in Fig. 9. For such high frequent switching schemes the distortions are mainly in the high frequent area and not taking place in the low frequent resonance area.
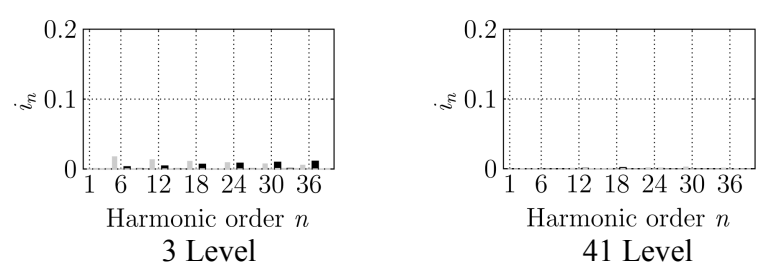

Fig. 9: SVPWM distortion spectra for 3 and 41 level CSI, 48 target points

The right side of Fig. 9 seems to indicate no harmonic content. But as already mentioned it can only not be seen, because it is taking place around the $48^{\text {th }}$ harmonic, cf. Fig. 7 (that can be seen at the THD, too).

\section{Conclusion}

Now which approach is feasible for the network of Fig. 1 and its behaviour shown in Fig. 2. The FFM avoids switching operations but seems to lead to a full six-pulse spectrum with similarly high harmonics even for a high level number (right side of Fig. 5). But those harmonics are quite small, the $-5^{\text {th }}$ is about $0.1 \%$ and the $+7^{\text {th }} 0.3 \%$. For high level numbers and an adequate choice of target points the SVPWM is able to operate with extremely small low order harmonics (right side of Fig. 7 and Fig. 9). Consequently both switching schemes could be used under certain circumstances.

The considerations also clarify why the switching schemes of CSI require more attention than those for a VSI. The resonance area has to be avoided which is a reason why the selective harmonic elimination methods are so widespread. So only more levels is due to practical reasons not the most useful answer. Rather a possibility to ensure that no low order harmonics occur and that does not make use of high frequent switching schemes and a large number of inverters. Such an approach could be to implement a CSI with real 12-pulse or approximated 18or 24-pulse behaviour. The 12-pulse behaviour for example could already be reached with only 3 standard CSI. This topology could then be switched with fundamental frequency but nevertheless avoid the resonance area of Fig. 2. Therefore these aspects will be the main focus of future research.

\section{References}

[1] H.-P. Nee and L. Ängquist, Perspectives on Power Electronics and Grid Solutions for Offshore Wind farms: Elforsk rapport 10:96. Available: http://www.elforsk.se/Rapporter/?download=repor t\&rid=10_96_(2013, Oct. 31).

[2] B. Wu, High-power converters and $A C$ drives. Hoboken, N.J, Chichester: John Wiley \& Sons, 2006.

[3] Z. Bai and Z. Zhang, "Conformation of Multilevel Current Source Converter Topologies Using the Duality Principle," IEEE Transactions on Power Electronics, vol. 23, no. 5, pp. 2260-2267, 2008.

[4] G. Mehlmann, R. Wendt, and G. Herold, "Space Vector Fundamental Frequency Modulation Compared with the Selective Harmonic Elimination Method," in Renewable Energy \& Power Quality Journal, European Association for the Development of Renewable Energies, Environment and Power Quality, Ed. 10th ed, 2012.

[5] G. Mehlmann, "Fundamental Frequency Modulation and Power Quality of MultilevelConverters" Thesis, Munich, 2013. 